\title{
Impact of plant-based diet on lipid risk factors for atherosclerosis
}

\author{
Agnieszka Kuchta ${ }^{1}$, Anna Lebiedzińska ${ }^{2}$, Marcin Fijałkowski ${ }^{3}$, Rafał Gałąska ${ }^{3}$, \\ Ewelina Kreft ${ }^{4}$, Magdalena Totoń ${ }^{1}$, Kuba Czaja ${ }^{2}$, Anna Kozłowska ${ }^{2}$, \\ Agnieszka Ćwiklińska ${ }^{1}$, Barbara Kortas-Stempak ${ }^{1}$, Adrian Strzelecki ${ }^{5}$, \\ Anna Gliwińska ${ }^{1}$, Kamil Dąbkowski ${ }^{1}$, Maciej Jankowski ${ }^{1}$ \\ ${ }^{1}$ Department of Clinical Chemistry, Medical University of Gdansk, Gdansk, Poland \\ ${ }^{2}$ Chair and Department of Bromatology, Medical University of Gdansk, Gdansk, Poland \\ ${ }^{3} 1^{\text {st }}$ Chair and Clinic of Cardiology, Medical University of Gdansk, Gdansk, Poland \\ ${ }^{4}$ Department of Monitored Therapy and Pharmacogenetics, \\ Medical University of Gdansk, Gdansk, Poland \\ ${ }^{5}$ Chair and Clinic of Internal Medicine, Connective Tissue Diseases and Geriatrics, \\ Medical University of Gdansk, Gdansk, Poland
}

\begin{abstract}
Background: The aim of the study was to investigate the effect of a vegan diet on the serum lipid profile with particular regard to the parameters characterizing the high-density lipoprotein (HDL) fractions in subjects without subclinical atherosclerosis, measured by carotid Doppler ultrasonography.

Methods and Results: Forty-two 23 to 38 year old subjects (21 omnivores and 21 vegans) participated in the study. Compared to the omnivores, the vegan subjects were characterized by lower parameters of lipid profile: total cholesterol $(p<0.001)$, low-density lipoprotein (LDL)-cholesterol ( $p<0.001)$, non-HDL-cholesterol $(p<0.001)$, apolipoprotein B (apoB) $(p<0.001)$ and phospholipids $(p<0.01)$. Concentration of HDL-cholesterol was apparently similar between groups. Furthermore, the parameters which characterize HDL particles (concentration of apolipoproteins AI [apoAI] and AII, HDL-phospholipids, LpAI fraction and pre$\beta 1-H D L$ fraction) were not significantly different between omnivore and vegan subjects. The apoB/apoAI ratio in vegans was lower than in omnivores $(p<0.01)$. There was no difference between serum concentration of triacylglycerols between omnivores and vegans. The activity of paraoxonase-1 and 8-iso-prostaglandin F2 $\alpha$ concentration were also not different between the study groups.

Conclusions: We suggest that a vegan diet may have a beneficial effect on serum lipid profile and cardiovascular protection, but it is not associated with changes in HDL composition. (Cardiol J 2016; 23, 2: 141-148)
\end{abstract}

Key words: atherosclerosis, plant-based diet, lipoproteins

Address for correspondence: Agnieszka Kuchta, MD, PhD, Department of Clinical Chemistry, Medical University of Gdansk, ul. Dębinki 7, 80-211 Gdańsk, Poland, e-mail: agakuchta@gumed.edu.pl

Received: 10.11.2015 Accepted: 27.11.2015 


\section{Introduction}

Atherosclerosis is one of the leading causes of death throughout the world [1]. A crucial role in the pathogenesis of atherosclerosis is played by abnormalities in plasma lipid and lipoprotein metabolism [2]. The levels of serum lipids and lipoproteins are sensitive to the type and amount of fat and cholesterol in the diet. It has been shown in animal and human studies that the intake of a high amount of saturated fats and cholesterol is atherogenic [2, 3].

The main source of saturated fats and cholesterol are products of animal origin, therefore it can be assumed that a vegan diet which excludes all products of animal origin could be beneficial in reducing the risk of atherosclerosis development. Indeed, some studies show that the use of a wellbalanced vegan diet can have a positive impact on cardiovascular risk $[4,5]$. The possible health benefit from a vegan diet may be related to the low intake of saturated fats, higher intake of plant food components such as vegetable oils, vegetable proteins, nuts, and fibers, which reduce serum lipids and may improve endothelial functions $[6,7]$. Of particular importance may also be a greater supply of phytosterols, plant derived compounds similar to cholesterol which have lipid-lowering properties. Phytosterols reduce absorption of cholesterol in the digestive tract and influence lipoprotein metabolism, which in turn may lead to a decrease in total cholesterol (TC) and low density lipoprotein (LDL) cholesterol (LDL-C) concentration [8, 9].

However, the impact of a vegan diet on other lipid-associated parameters is still ambiguous, for instance, a question still remains regarding the effect of a vegan diet on the quantity and quality of high density lipoproteins (HDL) [10]. HDL particles have an atheroprotective function and together with their main apolipoprotein, apolipoprotein $\mathrm{AI}$ (apoAI), play a key role in reverse cholesterol transport, removing excess cholesterol from peripheral tissues and delivering it to the liver. HDL particles also inhibit LDL oxidation, vascular inflammation and thrombosis. It is noteworthy that HDL particles are heterogeneous in nature and not all HDL particles may be equally protective [11]. Therefore, concentration of HDL cholesterol (HDL-C) is not a reliable indicator of the anti-atherogenic potential of HDL particles. This suggests further investigation aimed to characterize the quality of HDL particles such as pre$\beta 1$-HDL, a precursor form of HDL, or the amount of HDL fraction containing apoAI and deprived of
apoAII (LpAI) [12]. It also seems to be important to evaluate components of HDL other than cholesterol lipids, for example phospholipids [13].

It is also suggested that a vegan diet, despite the large quantities of herbal antioxidants, may increase the risk of oxidative stress due to an insufficient supply of vitamin B12 and increased levels of homocysteine, which in turn may increase the oxidative modification of lipids and risk of atherosclerosis [14].

The purpose of the present study was to investigate the protective role of a vegan diet on the serum lipid profile with particular regard to the parameters characterizing the HDL fractions in subjects without subclinical atherosclerosis, measured as carotid intima-media thickness (cIMT). We also investigated the effect of a vegan diet on the level of isoprostane (8-iso-prostaglandin $\mathrm{F} 2 \alpha$ ), a chemically stable lipid peroxidation product, and on the activity of paraoxonase-1 (PON-1), an HDL associated, antioxidant enzyme. Both of these parameters are not only oxidative balance markers, but have also been revealed as potential biofactors affecting the development of cardiovascular diseases $[15,16]$.

\section{Methods}

\section{Subjects}

All subjects participated voluntarily in the study and were 23 to 38 years old. Vegans were defined as those who do not eat any type of animal product, in other words, who exclude meat, dairy products and eggs at least from their diet. The subjects were on a vegan diet for at least 10 months (mean \pm standard deviation: $75 \pm 44$ ). All subjects before the start of a vegan diet had been on a vegetarian diet for at least 22 months (mean \pm standard deviation: $128 \pm 69$ ).

Omnivores were defined as people who eat food of both plant and animal origin. Dietary assessment was determined by investigators based on a self-reported questionnaire. Information on sociodemographic characteristics, such as sex, age, anthropometric data, physical activity, lifestyle, dietary habits, physician-diagnosed diseases, and medical history were obtained through interviews. All participants were non-smoking, sedentary or moderately physically active (at least 2 times a week). Eighty percent of vegans participating in the study received a vitamin supplementation at least ones daily. In the omnivores group, vitamins supplementation concerned $28 \%$ of respondents. The eclusion criteria were: current use of prescribed pharmaceuticals, intense physical activity 
(up to 4 times a week), and a body mass index (BMI) greater than $25 \mathrm{~kg} / \mathrm{m}^{2}$.

The Scientific and Ethics Committee of the Medical University of Gdansk approved the study, and an informed written consent was obtained from each participant after the design and the objective of the study were explained.

\section{Dietary assessment}

Subjects were interviewed and the average of the three $24 \mathrm{~h}$ dietary recalls were used to estimate daily consumption of different nutrients. Diet was assessed using a validated intervieweradministered quantitative food frequency questionnaire collecting information on portion size of commonly consumed food items. Standard portion size (e.g., tablespoon, ladle, and bowl expressed in [g]) and frequency (daily, weekly, monthly, never) were recorded. The energy value and contents of nutrients in daily food intake were appraised with the aid of a computer program (Diet-5) based on the current tables of values of nutritional products worked out at the National Food and Nutrition Institute in Warsaw, Poland [17]. The assessment of the participants' eating habits was carried out using Polish recommendations [18]. Energy and macronutrient intakes as well as vitamin intakes were calculated by multiplying the daily frequency of specific foods by a standard portion and by the amount of nutrient present in each gram.

\section{Clinical assessment and laboratory measurements}

Total cholesterol, triacylglycerols (TAG), and phospholipids (PL) were measured by standard enzymatic colorimetric tests (Pointe Scientific Poland) on a Multiskan ${ }^{\mathrm{TM}}$ GO Microplate Spectrophotometer (Thermo Scientific). HDLs were isolated by precipitation of apolipoprotein B (apoB)-containing lipoproteins with dextran sulfate $50000 / \mathrm{Mg}^{2+}$ reagent and HDL-C was determined enzymatically. LDL-C concentration was calculated using the Friedewald formula (LDL $=$ TC - HDL - TG/5). Non-HDL-cholesterol (nonHDL-C) was calculated according to the equation: nonHDL-C $=\mathrm{TC}-$ HDL-C.

Apolipoproteins B, AI and AII were assessed by nephelometric methods with antibodies from Dade Behring Marburg on a Behring laser nephelometer. LpAI was assayed by an electroimmunodiffusion (rocket) technique (Hydragel LpAI, Sebia, France). Pre- $\beta 1$-HDL levels were measured by a sandwich enzyme immunoassay using MAB55201 [19]. Serum 8-iso-prostaglandin F2 $\alpha$ concentration was measured by a sandwich enzyme-linked assay with a commercially available kit (Cayman Chemicals). PON1 activity was evaluated using two substrates: paraoxon for paraoxonase activity, and phenylacetate for arylesterase activity [20,21].

\section{Measurement of carotid artery intima-media thickness}

An experienced cardiologist, blinded to the subject diet, performed all cIMT measures. The cIMT measurement was performed with the patient in the supine position with the neck slightly extended and turned $45^{\circ}$ towards the opposite of the examined side to an $\mathrm{L} 11$ probe connected to a GE Vivid E9 BT12 cardiac ultrasound system. Right and left side common carotid artery images were obtained to measure cIMT by using three different angle views for each vessel: anterior, lateral and posterior. Optimal B-mode settings of gain, depth, focal zone placement, and compression were individually adjusted for each vessel to enhance arterial wall structures and image quality. cIMT was measured by a software package for automated border detection loaded on the system (EchoPAC, GE), and mean cIMT values were obtained.

\section{Statistical analysis}

Statistical analysis was performed using the Statistica software package (STATISTICA 10.0 Statsoft Poland). To establish data distributions, the Shapiro-Wilk test was used. Continuous variables were expressed as mean \pm standard deviation (SD) or median with $25^{\text {th }}$ and $75^{\text {th }}$ percentiles. Data analysis was performed using both parametric and non-parametric tests when appropriate. Comparison of unpaired data between the two groups was performed using the Student's t-test for Gaussian variables and the Mann-Whitney-U test for nonGaussian ones. The $p$ value obtained of less than 0.05 was considered statistically significant.

\section{Results}

Forty-two subjects between 23 and 38 years participated in the study; 21 omnivores and 21 vegans. The general and cardiovascular characteristics of study subjects are shown in Table 1 . The mean BMI and waist to hip ratio of omnivores and vegans were similar between groups and fell within the normal range. There was also no difference in systolic and diastolic blood pressure and heart rate between omnivore and vegan subjects. All measurements of cIMT in vegan subjects were in the 
Table 1. Baseline characteristics of the study groups.

\begin{tabular}{lccc}
\hline Variable & Omnivores $(\mathbf{n}=\mathbf{2 1})$ & Vegans $(\mathbf{n}=\mathbf{2 1})$ & $\mathbf{P}^{*}$ \\
\hline Gender: male/female & $9 / 12$ & $8 / 13$ & - \\
Age [years] & $28 \pm 4$ & $28 \pm 5$ & 0.928 \\
Body mass index $\left[\mathrm{kg} / \mathrm{m}^{2}\right]$ & $21.1 \pm 2$ & $22.3 \pm 2$ & 0.814 \\
Waist to hip ratio & $0.84 \pm 0.08$ & $0.83 \pm 0.08$ & 0.659 \\
Systolic BP $[\mathrm{mm} \mathrm{Hg}]$ & $123 \pm 13$ & $121 \pm 9$ & 0.565 \\
Diastolic BP $[\mathrm{mm} \mathrm{Hg]}$ & $75 \pm 10$ & $73 \pm 7$ & 0.457 \\
Heart rate [bmp] & $78 \pm 8$ & $80 \pm 7$ & 0.393 \\
IMT RCA [mm] & $0.55 \pm 0.07$ & $0.53 \pm 0.08$ & 0.394 \\
IMT LCA [mm] & $0.54 \pm 0.10$ & $0.52 \pm 0.09$ & 0.499 \\
\hline
\end{tabular}

Values are means \pm standard deviation; ${ }^{*}$ Student's t-test. BP — blood pressure; IMT — intima-media thickness; LCA — left carotid artery; $\mathrm{RCA}$ - right carotid artery

Table 2. Daily intake of selected nutrients and vitamins in omnivores and vegans (median values and interquartile ranges [IQR]).

\begin{tabular}{|c|c|c|c|c|c|c|c|}
\hline \multirow{2}{*}{$\begin{array}{l}\text { Daily intake of selected } \\
\text { nutrients }\end{array}$} & \multicolumn{3}{|c|}{ Omnivores $(n=15)$} & \multicolumn{3}{|c|}{ Vegans $(n=20)$} & \multirow[t]{2}{*}{$\mathbf{P}^{*}$} \\
\hline & Median & IQR range & DRls & Median & IQR range & DRls & \\
\hline Energy [kcal] & 1,914 & $1,528-2,306$ & $46^{\wedge}$ & 2,036 & $1,830-2,178$ & $15^{\wedge}$ & 0.962 \\
\hline Carbohydrate [g] & 218 & $193-285$ & $0^{\wedge}$ & 305 & $271-335$ & $10^{\wedge}$ & 0.004 \\
\hline Protein [g] & 82 & $62-97$ & $12^{\wedge}$ & 56 & $48-66$ & $10^{\wedge}$ & 0.003 \\
\hline Fiber $[\mathrm{g}]$ & 18 & $12-24$ & $75^{\wedge} \wedge$ & 34 & $29-39$ & $15^{\wedge} \wedge$ & 0.002 \\
\hline Fat $[\mathrm{g}]$ & 71 & $56-113$ & $0^{\wedge} \wedge \wedge$ & 72 & $53-81$ & $5^{\wedge \wedge \wedge}$ & 0.625 \\
\hline PUFAs [g] & 8 & $5-16$ & $68^{\wedge}$ & 15 & $11-18$ & $35^{\wedge}$ & 0.016 \\
\hline Vitamin A $[\mu \mathrm{g}]$ & 892 & $839-1,937$ & $12^{\wedge}$ & 1,512 & $903-1,733$ & $20^{\wedge}$ & 0.325 \\
\hline Vitamin $\mathrm{E}[\mu \mathrm{g}]$ & 10 & 6-14 & $37^{\wedge} \wedge$ & 17 & $12-29$ & $5^{\wedge \wedge}$ & 0.003 \\
\hline Vitamin $\mathrm{B}_{1}[\mu \mathrm{g}]$ & 1.4 & $0.9-2.5$ & $46^{\wedge}$ & 1.3 & $1.1-1.5$ & $28^{\wedge}$ & 0.805 \\
\hline Vitamin $\mathrm{B}_{2}[\mu \mathrm{g}]$ & 1.5 & $1.2-1.9$ & $25^{\wedge}$ & 1.4 & $1.0-2.9$ & $42^{\wedge}$ & 0.860 \\
\hline Vitamin $\mathrm{B}_{6}[\mu \mathrm{g}]$ & 2.1 & $1.4-3.2$ & $18^{\wedge}$ & 2.3 & $1.7-3.9$ & $10^{\wedge}$ & 0.421 \\
\hline Vitamin $\mathrm{B}_{12}[\mu \mathrm{g}]$ & 2.9 & $2.2-6.1$ & $6^{\wedge}$ & 44 & $11-93$ & $0^{\wedge}$ & $<0.001$ \\
\hline Vitamin $\mathrm{C}[\mu \mathrm{g}]$ & 84 & $79-159$ & $31^{\wedge}$ & 208 & $147-227$ & $5^{\wedge}$ & 0.014 \\
\hline
\end{tabular}

P values from Mann-Whitney $U$ tests for the assessment of significant differences between the median values; $\wedge$ Estimated average requirement;

$\wedge$ Adequate intake; $\wedge \wedge \wedge$ Dietary reference values; DRls - dietary reference intakes; PUFAs — polyunsaturated fatty acids

normal range, i.e. below $0.9 \mathrm{~mm}$, and did not significantly differ from omnivore subjects (Table 1).

The median values of the estimated intake of energy and macronutrients of the omnivores and vegans are shown in Table 2 . The total energy and fat intakes were similar for omnivores and vegans. The vegans consumed significantly more carbohydrates and the percentage of energy derived from carbohydrate in vegans was greater in comparison to the omnivores. However, $10 \%$ of vegan subjects were characterized by carbohydrate consumption under dietary reference intakes (DRI), but there were no omnivore subjects with carbohydrate consumption under DRI. Moreover, the vegan subjects consumed significantly more fibers and polyunsaturated fatty acids, but fewer proteins.

The median values of daily vitamin intake of both groups in comparison to DRIs are shown in Table 2 . The vegan subjects consumed significantly more vitamin $\mathrm{E}, \mathrm{B} 12$, and $\mathrm{C}$ than omnivore subjects. Although vitamin A, B1, B2 and B6 intake was apparently similar in both groups, a higher 

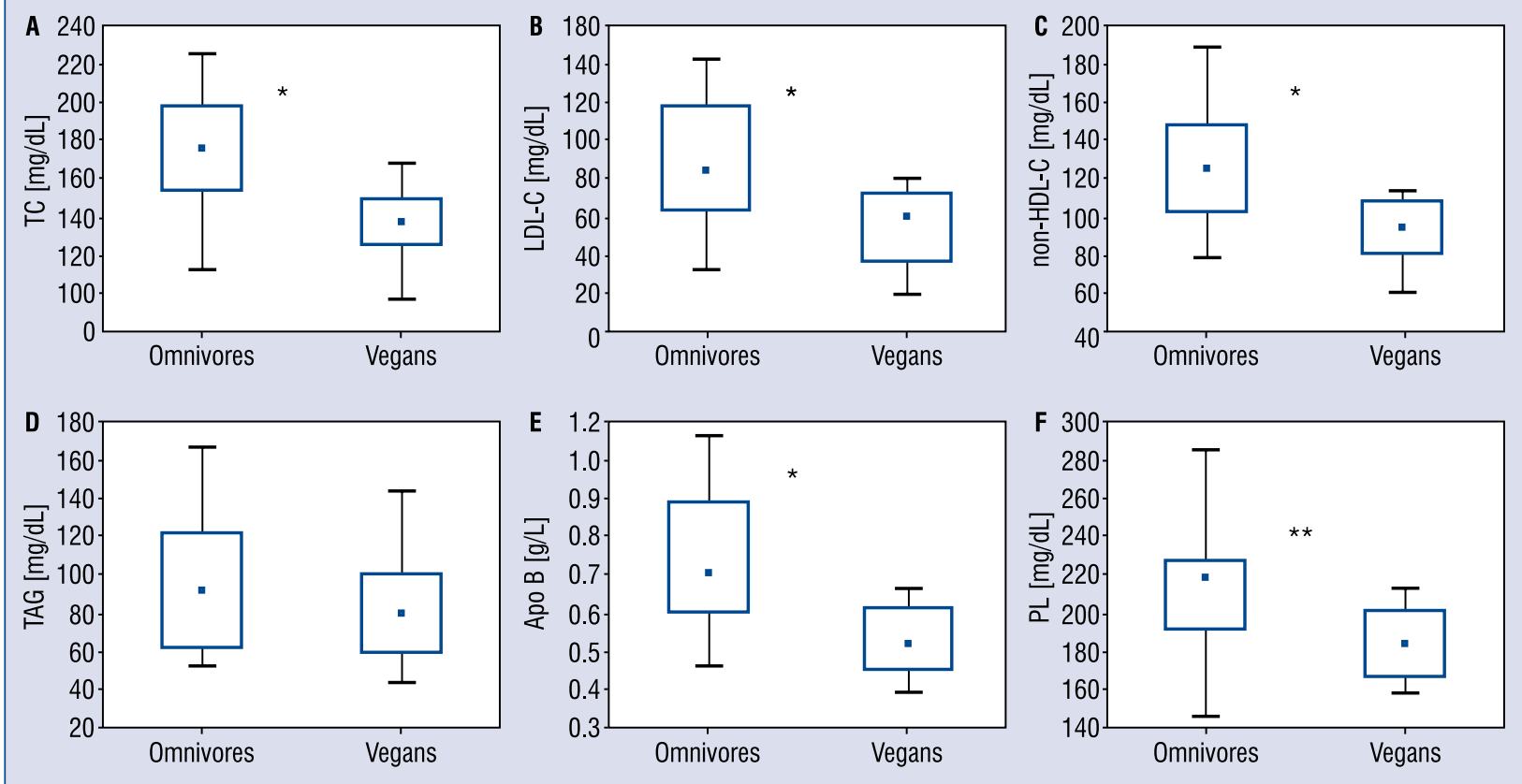

Figure 1. Serum concentrations of total cholesterol (TC; A), low-density lipoprotein cholesterol (LDL; B), non high density lipoprotein cholesterol (non-HDL-C; C), triacylglycerols (TAG; D), apolipoprotein B (apoB; E), phospholipids $(\mathrm{PL} ; \mathrm{F})$ in vegans and omnivores. Values are presented as median; 25-75 percentile; 5-95 percentile and assessed by Mann-Whitney U test; ${ }^{*} \mathrm{p}<0.001 ;{ }^{*} \mathrm{p}<0.01$.

Table 3. Serum concentrations of high-density lipoprotein parameters in omnivores and vegans (median values and interquartile ranges [IQR]).

\begin{tabular}{|c|c|c|c|c|c|}
\hline & \multicolumn{2}{|c|}{ Omnivores $(n=21)$} & \multicolumn{2}{|c|}{ Vegans $(n=21)$} & \multirow[t]{2}{*}{$P^{*}$} \\
\hline & Median & IQR range & Median & IQR range & \\
\hline $\mathrm{HDL}-\mathrm{C}[\mathrm{mg} / \mathrm{dL}]$ & 45 & $36-50$ & 45 & $42-52$ & 0.326 \\
\hline HDL-PL [mg/dL] & 90 & $77-98$ & 86 & $77-99$ & 1 \\
\hline ApoAI [g/L] & 1.6 & $1.5-2.1$ & 1.6 & $1.5-1.8$ & 0.542 \\
\hline ApoAll $[\mathrm{g} / \mathrm{L}]$ & 0.37 & $0.34-0.39$ & 0.38 & $0.32-0.39$ & 0.509 \\
\hline LpAI [g/L] & 0.53 & $0.36-0.75$ & 0.44 & $0.39-0.49$ & 0.188 \\
\hline $\operatorname{pre} \beta 1 \mathrm{HDL}[\mathrm{mg} / \mathrm{L}]$ & 37 & $29-47$ & 32 & $27-42$ & 0.294 \\
\hline
\end{tabular}

* $P$ values from Mann-Whitney $U$ tests for the assessment of significant differences between the median values; HDL-C - high density lipoprotein cholesterol; PL — phospholipids; apoAl and apoAll — apolipoproteins Al and All

percentage of the vegans consumed less vitamin A and vitamin $\mathrm{B} 2$ than the DRIs.

Figure 1 and Table 3 show serum lipid concentrations in omnivores and vegans. The vegan subjects were characterized by a lower concentration of TC (median: 140, range: $123-149$ vs. 173, 154-196 $\mathrm{mg} / \mathrm{dL}, \mathrm{p}<0.001$ ), LDL-C (median: 79, range: 68-91 vs. $100,87-133 \mathrm{mg} / \mathrm{dL}, \mathrm{p}<0.001)$, non-HDL-C (median: 98 , range: $81-108$ vs. $123,105-148 \mathrm{mg} / \mathrm{dL}$, $\mathrm{p}<0.001$ ), PL (median: 172, range: $165-194$ vs. 213, 186-224 mg/dL, $\mathrm{p}<0.01$ ), apoB (median: 0.54, range: $0.45-0.61$ vs. $0.69,0.61-0.83 \mathrm{~g} / \mathrm{L}, \mathrm{p}<0.001$ ), and apoB/apoA ratio (median: 0.31 , range: $0.26-0.40$ vs. $0.39,0.33-0.57, \mathrm{p}<0.01)$. Concentration of HDL-C was apparently similar between groups (Table 3 ). Additionally, the parameters which characterize HDL fraction (concentration of apolipoproteins AI and AII, HDL-PL, LpAI fraction, and pre- $\beta 1-\mathrm{HDL}$ fraction) were not significantly different between omnivore and vegan subjects (Table 3 ). There was also no difference between the serum concentration of TAG of the omnivores and vegans (Fig. 1). 
Table 4. Paraoxonase-1 (PON-1) activities and 8-iso-prostaglandin F2a (8-iso-PGF2a) concentrations in omnivores and vegans (median values and interquartile ranges [IQR]).

\begin{tabular}{|c|c|c|c|c|c|}
\hline \multirow[t]{2}{*}{ Variable } & \multicolumn{2}{|c|}{ Omnivores $(n=21)$} & \multicolumn{2}{|c|}{ Vegans $(n=21)$} & \multirow[t]{2}{*}{$\mathbf{P}^{*}$} \\
\hline & Median & IQR range & Median & IQR range & \\
\hline Paraoxonase activity [U/L] & 117 & $102-149$ & 123 & $108-150$ & 0.411 \\
\hline Arylesterease activity [kU/L] & 115 & $110-135$ & 122 & $92-136$ & 0.906 \\
\hline 8-iso-PGF2 $\alpha$ [pg/mL] & 114 & $91-150$ & 104 & $83-135$ & 0.526 \\
\hline
\end{tabular}

* $P$ values from Mann-Whitney $U$ tests for the assessment of significant differences between the median values

The activities of the antioxidant enzyme PON1, evaluated by paraoxonase and arylesterase activity, in omnivores and vegans are shown in Table 4 and were not different between both groups. The concentration of 8-iso-prostaglandin $\mathrm{F} 2 \alpha$, an index of oxidative stress, was also not different between both groups (Table 4).

\section{Discussion}

In the present study, we have provided evidence that the level and composition of HDL, an anti-atherogenic fraction of lipoproteins, are not affected in vegan subjects without subclinical atherosclerosis, suggesting that the beneficial effect of a vegan diet for cardiovascular protection is hardly associated with HDL protective ability. Vascular remodeling of the carotid artery, clinically defined as intima-media thickening (cIMT), is an important predictive phenotype for human cardiovascular disease. There were no significant differences in cIMT measurements between omnivores and vegans in our study, and what is more, all measurements were within a normal range that could be explained by the relatively young age of subjects.

From a nutrient intake perspective, the vegan diet of the study subjects was rich in carbohydrates, polyunsaturated fatty acids and dietary fiber but relatively low in proteins. Furthermore, no differences were detected when comparing the energy and fat intake of omnivores and vegans. This is reflected by similar BMI and waist to hip ratio in the investigated groups (Table 1).

Our results demonstrate that vegans, in comparison to omnivore subjects, had significantly lower concentrations of TC, LDL-C, non-HDL-C, $\mathrm{PL}$ and apoB. In terms of TC and LDL-C concentrations, this is well established because replacement of saturated fatty acids with polyunsaturated and monounsaturated fatty acids diminishes these parameters [22]. Additional higher contents of phytosterols in the vegan diet may cause a lipid- lowering effect [8]. Our study also confirms recent reports observed by Bradbury et al. [23] in the large European population which show positive results in reduction of apoB and also a lower concentration of non-HDL-C in the vegan group. However, they have not evaluated the concentrations of triglycerides and parameters associated with HDL particles [23].

A diet high in refined carbohydrates may increase TAG, whereas high fiber content appears to have opposite results. However, our study demonstrated that subjects following vegan diet do not have significantly lower triacylglycerol concentration compared with those following diets that include animal products. Reports about the effects of a plant-based diet on TAG concentration are unequivocal. In Barnard et al.'s study [24], a low fat vegetarian diet led to an increase in serum TAG. Similar results were obtained when investigating the vegan population of pre-menopausal women [10]. Other studies show a reduction in the level of TAG during a vegan diet [25]. In our study, we did not notice differences either in the concentrations of TAG or in the level TAG/HDL-C, which seems to be a more reliable predictor of myocardial infarctions [26, 27]. It is possible that other factors, e.g. environmental and genetic, may have an impact on TAG metabolism since it has been noticed that the effect of diet had greater benefits in subjects from developing countries [28].

HDL particles act as potential protectors against arterial disease mainly through their ability to promote cholesterol efflux from macrophages in the artery wall. However, HDLs have several additional protective properties that are independent of their involvement in cholesterol metabolism, among others they have properties that reduce oxidation, vascular inflammation and thrombosis. There is substantial evidence that the anti-atherogenic effect of HDL depends not only on its concentration expressed as HDL-C but also the quantitative and qualitative composition of the 
lipoproteins. Previously published results of studies do not provide a clear answer to the question of how, if at all, a vegan diet influences HDL particles. Some researchers have shown the lowering effects of a vegan diet on HDL-C concentration, which may impair its cardioprotective character $[10$, 29]. We did not find a significant lowering effect of vegan diet on HDL-C concentration (Table 3 ). Furthermore, we measured parameters other than HDL-C characterizing this fraction of lipoproteins. Concentrations of the apoAI and apoAII in vegan subjects were not different from these of omnivore subjects. Similarly, we did not notice differences in concentration of HDL particles containing apoAI but not apoAII (LpAI) between omnivores and vegans. Neither did we find significant differences in concentration of pre- $\beta 1-\mathrm{HDL}$, a precursor of HDL particles. Similar levels of major HDL apolipoproteins, LpAI fraction and pre- $\beta 1$-HDL fraction suggest that a vegan diet, in our study subjects, significantly affects neither serum HDL-C nor HDL composition. Some investigators postulated that the apoB/apoAI ratio may be useful in clinic as a convenient biomarker in metabolic syndrome predicting. We have shown that the apoB/apoAI ratio in vegan subjects was lower than in omnivores, confirming that a vegan diet may have a protective effect for metabolic syndrome development [30].

Metabolic syndrome and cardiovascular disorders are characterized by a disturbance in oxidative balance. It is suggested that a vegan diet as an extreme form of diet, despite the large quantities of herbal antioxidants, may increase the risk of oxidative stress due to the insufficient supply of vitamin B12 and increased levels of homocysteine, which in turn may increase the oxidative modification of lipids and risk of atherosclerosis [16]. The present data do not confirm this hypothesis, though this may result from different vitamin supplementation in the study populations. Analyzing the food intake questionnaire, we concluded that all vegan subjects included in our study used at least one vitamin preparation and daily intake of $\mathrm{E}, \mathrm{B} 12$, and $\mathrm{C}$ vitamins by our vegan group was higher than in omnivores. These results suggest that a vegan diet in our subjects meets their nutritional needs. This suggestion is supported by the results of PON-1 activity and concentration of isoprostane in blood. We did not notice the effects of diet on PON-1 activity either in paraoxonase or in aryloesterase activity. We also showed no differences in 8 -iso-prostaglandin $\mathrm{F} 2 \alpha$ concentration, which is a sensitive parameter of oxidative stress and lipid peroxidation.

\section{Conclusions}

In conclusion, the findings of this study have shown that a vegan diet did not change the parameters expressly connected with HDL fraction. We also did not show the effect of diet on lipoprotein peroxidation markers. Lower concentration of TC, LDL-C as also a lower ratio of apoB/apoAI suggest that there could be a beneficial effect of a vegan diet for cardiovascular protection. However, because vegan diet is an extreme form of diet we should be aware that its long-term effect on health is dependent upon adequate delivery of all the needed micronutrients which can be a serious challenge.

\section{Acknowledgments}

This project was supported by the Ministry of Science and Higher Education of the Republic of Poland, from the quality-promoting subsidy, under the Leading National Research Centre (KNOW) program for the period of 2012-2017.

\section{Conflict of interest: None declared}

\section{References}

1. Weber C, Noels H. Atherosclerosis: Current pathogenesis and therapeutic options. Nat Med, 2011; 17: 1410-1422.

2. Schaefer EJ. Lipoproteins, nutrition, and heart disease. Am J Clin Nutr, 2002; 75: 191-212.

3. Ye P, Cheah IK, Halliwell B. High fat diets and pathology in the guinea pig. Atherosclerosis or liver damage? Biochim Biophys Acta, 2013; 1832: 355-364.

4. Barnard ND, Cohen J, Jenkins DJ et al. A low-fat vegan diet improves glycemic control and cardiovascular risk factors in a randomized clinical trial in individuals with type 2 diabetes. Diabetes Care, 2006; 29: 1777-1783.

5. Jenkins DJ, Kendall CW, Popovich DG et al. Effect of a very-highfiber vegetable, fruit, and nut diet on serum lipids and colonic function. Metabolism, 2001; 50: 494-503.

6. Rao V, Al-Weshahy A. Plant-based diets and control of lipids and coronary heart disease risk. Curr Atheroscler Rep, 2008; 10: 478-485.

7. Casiglia E, Tikhonoff V, Caffi S et al. High dietary fiber intake prevents stroke at a population level. Clin Nutr, 2013; 32: 811-818.

8. Racette SB, Spearie CA, Phillips KM, Lin X, Ma L, Ostlund RE. Phytosterol-deficient and high-phytosterol diets developed for controlled feeding studies. J Am Diet Assoc, 2009; 109: 2043-2051.

9. Ostlund RE. Phytosterols, cholesterol absorption and healthy diets. Lipids, 2007; 42: 41-45.

10. Huang YW, Jian $\mathrm{ZH}$, Chang $\mathrm{HC}$ et al. Vegan diet and blood lipid profiles: A cross-sectional study of pre and postmenopausal women. BMC Womens Health, 2014; 14: 55.

11. Navab M, Reddy ST, Van Lenten BJ, Fogelman AM. HDL and cardiovascular disease: Atherogenic and atheroprotective mechanisms. Nat Rev Cardiol, 2011; 8: 222-232.

12. Guey LT, Pullinger CR, Ishida BY et al. Relation of increased prebeta-1 high-density lipoprotein levels to risk of coronary heart disease. Am J Cardiol, 2011; 108: 360-366. 
13. Piperi C, Kalofoutis C, Papaevaggeliou D, Papapanagiotou A, Lekakis J, Kalofoutis A. The significance of serum hdl phospholipid levels in angiographically defined coronary artery disease. Clin Biochem, 2004; 37: 377-381.

14. Krajcovicová-Kudlácková M, Blazícek P, Babinská K, Kopcová J, Klvanová J, Béderová A, Magálová T. Traditional and alternative nutrition: Levels of homocysteine and lipid parameters in adults. Scand J Clin Lab Invest, 2000; 60: 657-664.

15. de Faria AP, Fontana V, Modolo R et al. Plasma 8-isoprostane levels are associated with endothelial dysfunction in resistant hypertension. Clin Chim Acta, 2014; 433: 179-183.

16. Bayrak A, Bayrak T, Tokgözoglu SL et al. Serum pon-1 activity but not q192r polymorphism is related to the extent of atherosclerosis. J Atheroscler Thromb, 2012; 19: 376-384.

17. Kunachowicz H, Nadolna I, Przygoda B, Iwanow K. Tables of nutritionalvalue of food products and dishes, 3rd edition extended and updated. National Food and Nutrition Institute, Warsaw, Poland 2010.

18. Jarosz M. Normy żywienia dla populacji polskiej: Nowelizacja. National Food and Nutrition Institute, Warsaw, Poland 2012.

19. Miyazaki O, Kobayashi J, Fukamachi I, Miida T, Bujo H, Saito Y. A new sandwich enzyme immunoassay for measurement of plasma pre-beta1-hdl levels. J Lipid Res, 2000; 41: 2083-2088.

20. MacKness B, Mackness MI, Durrington PN et al. Paraoxonase activity in two healthy populations with differing rates of coronary heart disease. Eur J Clin Invest, 2000; 30: 4-10.

21. Nakanishi M, Takanami Y, Maruyama T et al. The ratio of serum paraoxonase/arylesterase activity using an improved assay for arylesterase activity to discriminate pon1(r192) from pon1(q192). J Atheroscler Thromb, 2003; 10: 337-342.

22. Siri-Tarino PW, Sun Q, Hu FB, Krauss RM. Saturated fatty acids and risk of coronary heart disease: Modulation by replacement nutrients. Curr Atheroscler Rep, 2010; 12: 384-390.
23. Bradbury KE, Crowe FL, Appleby PN, Schmidt JA, Travis RC Key TJ. Serum concentrations of cholesterol, apolipoprotein a-i and apolipoprotein b in a total of 1694 meat-eaters, fisheaters, vegetarians and vegans. Eur J Clin Nutr, 2014; 68: 178$-183$.

24. Barnard ND, Scialli AR, Bertron P, Hurlock D, Edmonds K, Talev L. Effectiveness of a low-fat vegetarian diet in altering serum lipids in healthy premenopausal women. Am J Cardiol, 2000; 85: 969-972.

25. Toohey ML, Harris MA, DeWitt W, Foster G, Schmidt WD, Melby CL. Cardiovascular disease risk factors are lower in african-american vegans compared to lacto-ovo-vegetarians. J Am Coll Nutr, 1998; 17: 425-434

26. Cordero A, Andrés E, Ordoñez B et al.; Investigators MSASS: Usefulness of triglycerides-to-high-density lipoprotein cholesterol ratio for predicting the first coronary event in men. Am J Cardiol, 2009; 104: 1393-1397.

27. Shimizu Y, Nakazato M, Sekita T et al. Association between hemoglobin and diabetes in relation to the triglycerides-to-highdensity lipoprotein cholesterol (TG-HDL) ratio in Japanese individuals: The Nagasaki Islands Study. Intern Med, 2014; 53: 837-843.

28. Zhang Z, Ma G, Chen S et al. Comparison of plasma triacylglycerol levels in vegetarians and omnivores: A meta-analysis. Nutrition, 2013; 29: 426-430.

29. Waldmann A, Koschizke JW, Leitzmann C, Hahn A. German vegan study: Diet, life-style factors, and cardiovascular risk profile. Ann Nutr Metab, 2005; 49: 366-372.

30. Jing F, Mao Y, Guo J et al. The value of apolipoprotein b/apolipoprotein a1 ratio for metabolic syndrome diagnosis in a Chinese population: A cross-sectional study. Lipids Health Dis, 2014; 13: 81 . 